\title{
Electroacupuncture improves myocardial ischemia injury via activation of adenosine receptors
}

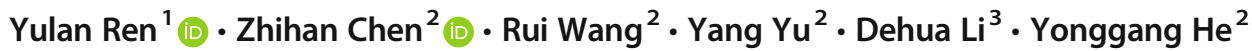

Received: 3 April 2020 / Accepted: 17 May 2020 / Published online: 6 July 2020

(C) The Author(s) 2020

\begin{abstract}
Electroacupuncture (EA) can improve myocardial ischemia (MI) injury; nevertheless, the mechanism is not entirely clear. And there were disagreements about whether the effect of EA at acupoint in disease-affected meridian is better than EA at acupoint in non-affected meridian and sham acupoint. Here, we showed that the effect of EA at Neiguan (PC6) is better than EA at Hegu (LI4) and sham acupoint in affecting RPP and ECG, increasing ATP and ADO production, decreasing AMP production, and upregulating the mRNA expression levels of A1AR, A2aAR, and A2bAR; knockdown of A1AR or A2bAR reversed the effect of EA at PC6 in alleviating MI injury; knockdown of A2aAR had no influence on the cardiac protection of EA at PC6; thus, the cardioprotective effect of EA at PC6 needs A1AR and A2bAR, instead of A2aAR; considering that the cardio protection of adenosine receptor needs activation of other adenosine receptors, one of the reasons may be that after silence of A1AR or A2bAR, EA at PC6 could not impact the expression levels of the other two adenosine receptors, and after silence of A2aAR, EA at PC6 could impact the expression levels of A1AR and A2bAR. These results suggested that EA at PC6 may be a potential and effective treatment for MI by activation of A1AR and A2bAR.
\end{abstract}

Keywords Acupuncture $\cdot$ Myocardial ischemia $\cdot$ A1 adenosine receptor $\cdot$ A2a adenosine receptor $\cdot$ A2b adenosine receptor

\section{Introduction}

Myocardial ischemia (MI) is one of heart conditions caused by lack of coronary blood flow [1], which is the primary cause of ischemic heart disease (IHD). The "The top 10 causes of death" indicated that IHD is one of the biggest killers in the world, accounting for around 9 million death in 2016 [2]. "Guideline on the Assessment and Management of
Cardiovascular Risk in China," which was published in 2019, showed that cardiovascular disease is the leading cause of death and burden of disease in China, and over 1 million people died of coronary heart disease [3]. In addition, IHD burden the economy $[3,4]$. Currently, the primary therapeutic methods include percutaneous coronary intervention, coronary artery bypass grafting, and medical therapy [5-11]. On account of the efficacy and safety of acupuncture, increasing

Yulan Ren and Zhihan Chen contributed equally to this work.

Yulan Ren

ryl@cdutcm.edu.cn

Zhihan Chen

czhcoral@hotmail.com

Rui Wang

13371567991@163.com

Yang $\mathrm{Yu}$

zj110110@163.com

Dehua Li

si_ff@163.com
Yonggang He

HYG6292@163.com

1 School of Chinese Classics, Chengdu University of Traditional Chinese Medicine, No. 37, Shierqiao Road, Jinniu District, Chengdu, Sichuan, China

2 School of Acupuncture Moxibustion and Tuina, Chengdu University of Traditional Chinese Medicine, Chengdu, Sichuan, China

3 Clinical Medical College, Chengdu University of Traditional Chinese Medicine, Chengdu, Sichuan, China 
patients are willing to receive acupuncture treatment. There is long history of application of acupuncture in improving MI. Previous studies indicated that acupuncture is effective in alleviating MI [12-15], and Neiguan (PC6) is the most frequently used acupoint [16]. The effect of acupuncture in MI was verified; nevertheless, there are different results of the effect of acupoint in disease-affected meridian, acupoint in nonaffected meridian, and sham acupoint [12, 17, 18]. Previous animal experiments were conducted to reveal mechanism of acupuncture in improving MI [13, 19, 20]. Some experiments showed that acupuncture could impact the express level of A1 adenosine receptor (A1AR) or $\mathrm{A} 2 \mathrm{a}$ adenosine receptor (A2aAR) or A2b adenosine receptor (A2bAR) [21, 22]. However, it cannot verify that the effect of acupuncture in MI depends on A1AR, A2aAR, and A2bAR. Thus, we hypothesized that acupoint in disease-affected meridian might be more effective than acupoint in non-affected meridian and sham acupoint; the effect of acupuncture at acupoint in disease-affected meridian in improving MI may depend on A1AR or A2aAR or A2bAR. To test these hypotheses, this experiment was conducted to evaluate (a) the differences in the effect of acupoint in disease-affected meridian (PC6), acupoint in non-affected meridian (Hegu (LI4)), and sham acupoint; (b) the roles of A1AR, A2aAR, and A2bAR in the effect of acupuncture at PC6; and (c) the possible reason for the results that the effect of acupuncture at PC6 depends on one of adenosine receptors.

\section{Materials and methods}

\section{Subjects}

Male and female Sprague-Dawley (SD) rats (Charles River, Beijing, China), weighing 200-220 g, received ad libitum food and water, and were housed in a temperature-controlled room $\left(25 \pm 3{ }^{\circ} \mathrm{C}\right)$ under a 12-h light/dark cycle. This study was conducted in accordance with the Guide for the Care and Use of Laboratory Animals (National Academies Press) and was approved by ethical committee of Chengdu University of Traditional Chinese Medicine (no. 2014-03).

\section{Reagents}

Recombinant virus plasmid and the carriers of auxiliary packaging original plasmids were purchased from Shanghai Genechem Co., Ltd. (Shanghai, China). Adora1, adora2a, and adora $2 \mathrm{~b}$ mRNA in situ hybrization kits were purchased from Boster Biological Technology Co., Ltd. (CA, USA). 293 T cell was purchased from Cell Resource Center of Shanghai Institutes for Biological Sciences (Shanghai, China).

\section{Grouping}

After 1-week acclimatization, 140 rats were randomly divided equally into 14 groups, namely normal control (NC) group, sham operation (SO) group, model (M) group, Neiguan (N) group, Hegu (H) group, sham acupoint (SA) group, negative lentivirus infected (NLI) group, A1AR lentivirus-infected (A1LI) group, A2aAR lentivirus-infected (A2aLI) group, A2bAR lentivirus-infected (A2bLI) group, negative lentivirus-infected Neiguan (NLIN) group, A1AR lentivirusinfected Neiguan (A1LIN) group, A2aAR lentivirus-infected Neiguan (A2aLIN) group, and A2bAR lentivirus-infected Neiguan (A2bLIN) group. In NC group, rats received no operation; in SO group, rats were only threaded, but not ligated left anterior descending coronary artery (LADCA); in M group, rats received ligation of LADCA; in $\mathrm{N}$ group, rats received ligation of LADCA and electroacupuncture (EA) treatment at bilateral PC6; in $\mathrm{H}$ group, rats received ligation of LADCA and EA treatment at bilateral LI4; in SA group, rats received ligation of LADCA and EA treatment at bilateral sham acupoint; in NLI, A1LI, A2aLI, and A2bLI groups, rats received corresponding lentivirus injection and ligation of LADCA; in NLIN, A1LIN, A2aLIN, and A2bLIN groups, rats received corresponding lentivirus injection, ligation of LADCA, and EA treatment at bilateral PC6.

\section{MI injury}

The rats were anesthetized with $10 \%$ chloral hydrate $(0.4 \mathrm{ml} /$ $100 \mathrm{~g} \mathrm{IP}$ ) and were positioned on an operating table for small animals (Fig. 1). After a thoracotomy performed in the fourth intercostal space, the LADCA was ligated 2 to $3 \mathrm{~mm}$ from its origin between the pulmonary artery conus and the left atrium with 6-0 silk. Occlusion of LADCA was verified by regional color change in the myocardial surface local and distal to the ligation, and change in electrocardiography (ECG). For M group, the LADCA of rats were only threaded with $6-0$ silk.

\section{Lentivirus infection}

The rats were anesthetized by intraperitoneal injection of chloral hydrate and received above thoracotomy. After exposure of heart, $50 \mu \mathrm{l}$ corresponding lentivirus solution $\left(1 \times 10^{7} \mathrm{TU} /\right.$ $100 \mu \mathrm{l})$ was injected into left ventricle anterior wall at four different points. Finally, the chest was closed.

\section{EA treatment}

The rats received EA treatment a day after MI operation. Each EA treatment lasted $20 \mathrm{~min}$; the rats received 5 times of treatment once every day for 5 consecutive days. Once-off stainless steel acupuncture needle (Suzhou Hualun Medical Appliance Co., Ltd., Jiangsu, China) was inserted to a depth 


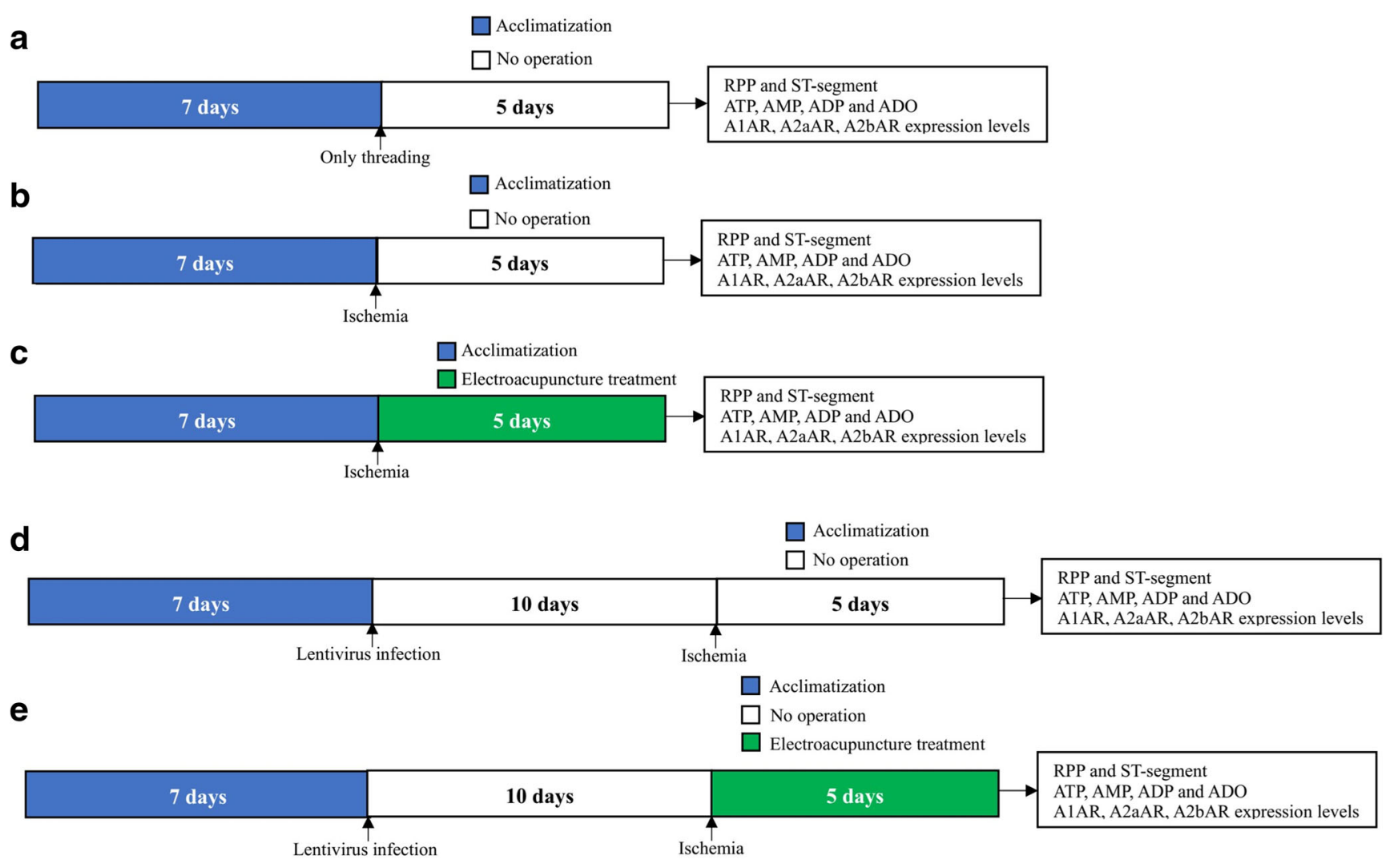

Fig. 1 Flow chart of the study protocol. a SO group. b M group. $\mathbf{c}$ N, H, and SA group. d NLI, A1LI, A2aLI, and A2bLI group. e NLIN, A1LIN, A2aLIN, and A2bLIN group

of 1-2 $\mathrm{mm}$. The acupuncture needle was connected to the HANS Acupuncture Point Nerve Stimulator (Nanjing Jisheng Medical Technology Co., Ltd., Jiangsu, China). The frequency was set at $2 / 100 \mathrm{~Hz}$, and the intensity of stimulation was $0.1-1 \mathrm{~mA}$. The procedures of all EA groups $(\mathrm{N}, \mathrm{H}, \mathrm{SA}$, NLIN, A1LIN, A2aLIN, A2bLIN groups) were the same. The location of acupoints, which refers to previous literatures [23, 24], is as following: (1) PC6: on the anteromedial aspect of the forelimb, between the ulna and the radius, $3 \mathrm{~mm}$ proximal to the wrist joints; (2) LI6: on forelimb, between the first metacarpal bone and the second metacarpal bone; and (3) sham acupoint: on the dorsal aspect of the forelimb, the spatia between the third metatarsal bone and the fourth metatarsal bone.

\section{Determination of rate pressure product and ST segment}

Left ventricular developed pressure (LVDP) and heart rate (HR) obtained by Data Acquisition System (iWorx, IL, USA) were used to calculate rate pressure product (RPP), according to the previously described method [25]: RPP = LVDP $\times$ HR. Data Acquisition System was also adopted to record ECG. All rats were calculated RPP and were recorded ECG before taking sample.

\section{High-performance liquid chromatography}

HPLC was used to determine the production of ATP, AMP, ADP, and ADO in myocardial tissue. The samples were determined by $2 \mathrm{~mol} / 1 \mathrm{HClO}_{4}(20 \mu \mathrm{l} / \mathrm{mg})$. After centrifugation at $4{ }^{\circ} \mathrm{C}$ and $20,000 \times g$ for $10 \mathrm{~min}$, aliquots of the supernatant were collected. To collect supernatant to regulate $\mathrm{PH}$ value to $6.5-7.0,1 \mathrm{~mol} / 1 \mathrm{~K}_{2} \mathrm{CO}_{3}$ was added. After blending, the mixture was stood on ice for $5 \mathrm{~min}$. After centrifugation at $4{ }^{\circ} \mathrm{C}$ and $20,000 \times g$ for $10 \mathrm{~min}$, the supernatant was collected for HPLC determination of ATP, AMP, ADP, and ADO concentrations. The chromatographic conditions were as follows: (1) chromatographic column: hypersil C18, $5 \mu \mathrm{m}, 4.6 \times 200 \mathrm{~mm}$; (2) mobile phase: $0.2 \mathrm{~mol} / 1$ phosphate potassium salt buffer (pH 6.0); and (3) flow rate $0.9 \mathrm{ml} / \mathrm{min}$. Ultraviolet-visible detector (Dalian Elite Analytical instrument Co., Ltd., Liaoning, China) was adopted to carry out chromatographic analysis.

\section{In situ hybrization}

Cardiac tissue was fixed in $4 \%$ paraformaldehyde overnight and was soaked in phosphate buffer solution (PBS) 4-5 times for $5 \mathrm{~min}$. Cardiac tissue was put into $30 \%$ sucrose $/ 0.1 \mathrm{M}$ 
PBS. Two days later, cardiac slice was soaked in 0.1 M PBS (once for $5 \mathrm{~min}$ ), $0.1 \mathrm{M}$ glycine/0.1 M PBS (once for $5 \mathrm{~min}$ ), 0.3\% Triton X-100/0.1 M PBS (once for $10 \mathrm{~min}$ ), and $0.1 \mathrm{M}$ PBS (three times for $5 \mathrm{~min}$ ). Fifty microliter proteinase was added to incubate at $37{ }^{\circ} \mathrm{C}$ for $30 \mathrm{~min}$. Cardiac slice was soaked in $4 \%$ paraformaldehyde for $5 \mathrm{~min}$ and was washed by $0.1 \mathrm{M}$ PBS twice for $5 \mathrm{~min}$. Cardiac slice was put into $0.25 \%$ acetic anhydride $/ 0.1 \mathrm{M}$ triethanolamine for $10 \mathrm{~min}$. Cardiac slice was pre-hybridized by pre-hybridization solution at $42{ }^{\circ} \mathrm{C}$ for $30 \mathrm{~min}$ and was hybridized by hybridization solution at $42{ }^{\circ} \mathrm{C}$ overnight. Four times SSC, $2 \times \mathrm{SSC}, 1 \times \mathrm{SSC}$, $0.5 \times \mathrm{SSC}, 0.2 \times \mathrm{SSC}, 0.2 \times \mathrm{SSC} / 0.1 \mathrm{M} \mathrm{PBS}, 0.05 \mathrm{M} \mathrm{PBS}$ were, respectively, used to wash cardiac slice. Then, cardiac slice was coated by $3 \% \mathrm{BSA} / 0.05 \mathrm{M} \mathrm{PBS}$ at $37{ }^{\circ} \mathrm{C}$ for $30 \mathrm{~min}$. Anti-digoxin/antiserum alkaline phosphatase was added to cardiac slice, and cardiac slice was, respectively, washed by 0.05 M PBS, TSM1, and TSM2. ImagePro Plus 6.0 software (Media Cybernetics, Inc., MD, the USA) was adopted to analyze results.

\section{Design and construction of lentivirus RNA interference vector}

Short hairpin RNA (shRNA) interference sequences were designed for A1AR, A2aAR, and A2bAR (accession no. NM_017155, NM_053294, and NM_017161) to construct recombinant shuttle plasmids and packaging plasmids (pFUGW-RNAi). The target sequences for A1AR, A2aAR, A2bAR, and negative control are as follows: 5'-CTTC TTTGCGTTCGTGTTA-3', 5'-GATTTGGAATGACC ACTTC-3', 5'-GTGTCTCTTTGAGAACGTA-3', 5'-TTCT CCGAACGTGTCACGT-3'. 293 T cells were transfected by sequenced plasmids and transfection reagent RNA-mate. The supernatants with lentivirus particles were concentrated and purified.

\section{Statistical analysis}

Statistical analysis was made using SPSS 26.0 software (IBM, $\mathrm{NY}$, USA). All data were expressed as mean $\pm \mathrm{SD}$ and were analyzed using one-way ANOVA with post hoc Tukey tests or Games-Howell tests. Differences were considered statistically significant when $P$ values were less than 0.05 .

\section{Results}

\section{Effects of EA on RPP and ST segment}

As shown in Fig. 1a, b, the value of RPP was significantly reduced and ST segment was significantly elevated in the $\mathrm{M}$ group compared with the $\mathrm{NC}$ group and the $\mathrm{SO}$ group (all $P<0.01$ ); thus, MI injury model is successful.
Compared with the M group, the value of RPP was significantly increased and ST segment was depressed in the $\mathrm{N}$ group (Fig. 2a, b, all $P<0.01$ ) and the value of RPP and ST segment had no significant change in the $\mathrm{H}$ group and the SA group (Fig. 2a, b, all $P>0.05$ ). Compared with the $\mathrm{N}$ group, the value of RPP was reduced and ST segment were elevated in the $\mathrm{H}$ group and the SA group (Fig. 2a, b, all $P<0.01$ ).

In addition, compared with the NLI group, the value of RPP was increased and ST segment was depressed in the NLIN group (Fig. 2c, d, all $P<0.01$ ); compared with the A1LI group, the value of RPP and ST segment in the NLIN group had no significant change (Fig. 2c, d, all $P>0.05$ ); compared with the A2aLI group, the A2aLIN group had significantly increase the value of RPP and depress ST segment (Fig. 2c, d, all $P<0.01$ ); compared with the A2bLI group, the A2bLIN group had no significant change in the value of RPP and ST segment (Fig. 2c, d, all $P>0.05$ ).

\section{Effects of EA on ATP, AMP, ADP, and ADO concentrations}

The results showed that MI injury caused decrease in the concentrations of ATP and ADO and increase in the concentration of AMP (Fig. 3a-d, all $P<0.01$ ) and no change in the concentration of ADP (Fig. 3c, all $P>0.05$ ) compared with the $\mathrm{NC}$ group and the SO group. EA treatment at PC6 significantly increased the concentrations of ATP and ADO, and decreased the concentration of AMP (Fig. 3a-d, all $P<0.01$ ); however, EA treatment at PC6 did not significantly impacted the concentration of ADP (Fig. 3c, $P>0.05$ ). EA treatment at LI6 and sham acupoint had no significant impact on the concentrations of ATP, AMP, ADP, and ADO (Fig. 3a-d, all $P>0.05$ ). Compared with the $\mathrm{H}$ group and the SA group, the concentrations of ATP and ADO were increased, and the concentration of AMP was reduced by EA treatment at PC6 (Fig. 3a-d, all $P<0.01$ ), and the concentration of ADP was not impacted (Fig. 3c, $P>0.05$ ).

Figure $3 \mathrm{e}-\mathrm{h}$ show that the concentrations of ATP and ADO have significantly risen and the concentration of AMP is decreased in the NLI group (all $P<0.01$ ), and the concentration of ADP is not different from that of the NLI group $(P>0.05)$; the A1LI group and the A1LIN group have no statistical difference in the concentrations of ATP, AMP, ADP, and ADO (all $P>0.05$ ); the concentrations of ATP and ADO are significantly increased, and the concentration of AMP is decreased (all $P<0.01$ ), and the concentration of ADP is not changed $(P>0.05)$ in the A2aLIN group compared with the A2aLI group; the A2bLI group and the A2bLIN group also have no significant difference in the concentrations of ATP, AMP, $\mathrm{ADP}$, and ADO (all $P>0.05$ ). 

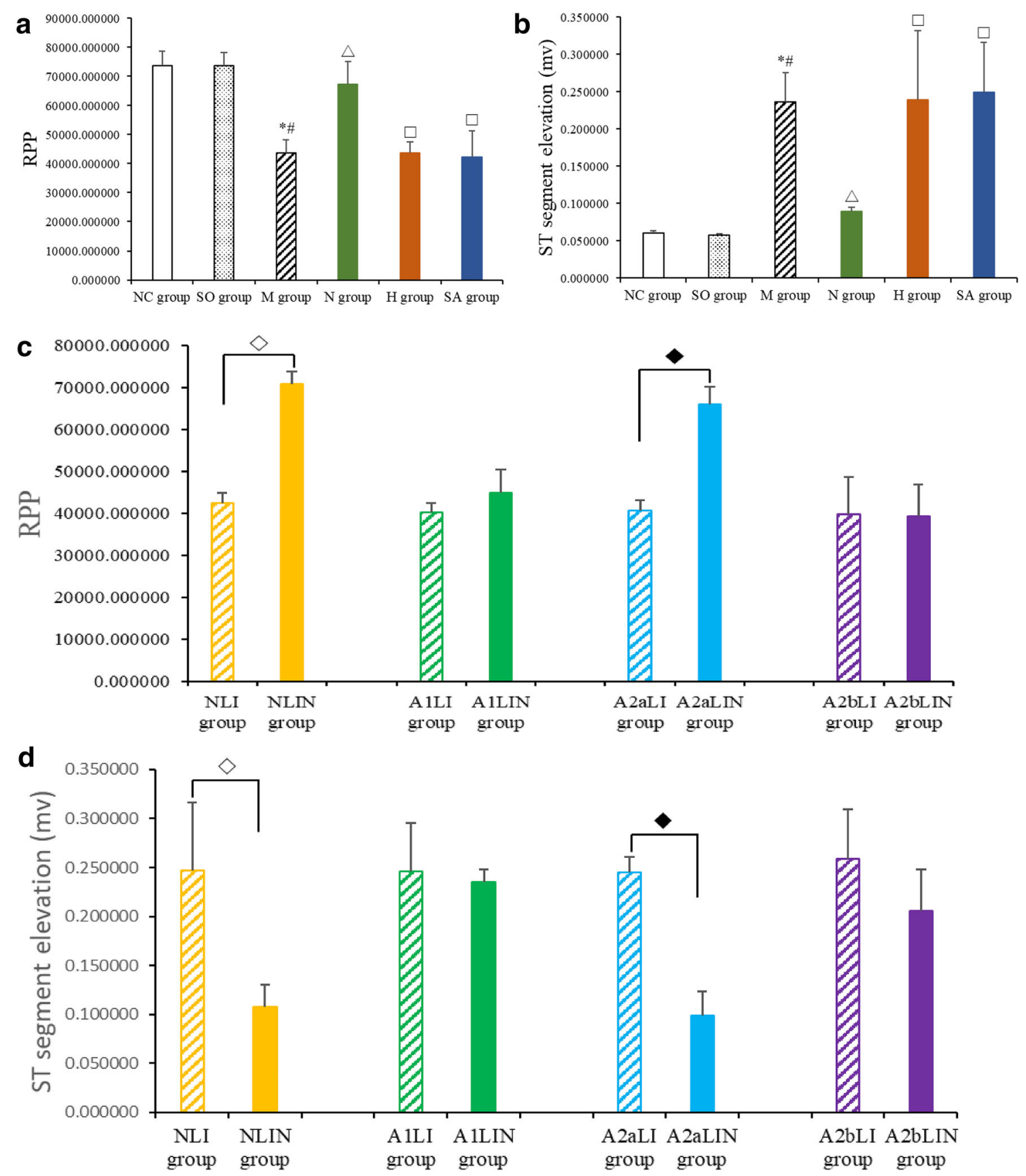

Fig. 2 Effects of EA on RPP and ST segment. a, $\mathbf{c}$ Rate pressure product (RPP) was calculated by left ventricular developed pressure (LVDP) and heart rate (HR). EKG was recorded from II limb leads with recorder speed $50 \mathrm{~ms} / \mathrm{div}$, and ST segment alterations were dedicated in $\mathbf{b}$ and $\mathbf{d}$ after

5 days followed by surgical procedure. Data are presented as mean $\pm \mathrm{SD}$. $* P<0.01$ versus NC group, $\# P<0.01$ versus SO group, $\triangle P<0.01$ versus M group, $\diamond P<0.01$ versus NLI group, $\bullet P<0.01$ versus A2aLI group

\section{Effects of EA on the mRNA expression levels of A1AR, A2aAR, and A2bAR}

Compared with the NC group and the SO group, the mRNA expression levels of A1AR, A2aAR, and A2bAR were significantly upregulated in the $\mathrm{M}$ group (Fig. $4 \mathrm{a}-\mathrm{c}$, all $P<0.01$ ). EA treatment at PC6 could significantly upregulate the mRNA expression levels of A1AR, A2aAR, and A2bAR (Fig. 4a-c, all $P<0.01$ ); however, EA treatment at LI6 and sham acupoint could not significantly upregulate the mRNA expression levels of A1AR, A2aAR, and A2bAR (Fig. 4a-c, all $P>0.05$ ). The mRNA expression levels of A1AR, A2aAR, and $\mathrm{A} 2 \mathrm{bAR}$ were higher in the $\mathrm{N}$ group than those of the $\mathrm{H}$ group and the SA group (Fig. $4 \mathrm{a}-\mathrm{c}$, all $P<0.01$ ).

The mRNA expression levels of A1AR, A2aAR, and A2bAR in the NLIN group were higher than those of the NLI group (Fig. 4d-f, all $P<0.01$ ); no significant differences were observed in the mRNA expression levels of A2aAR and 

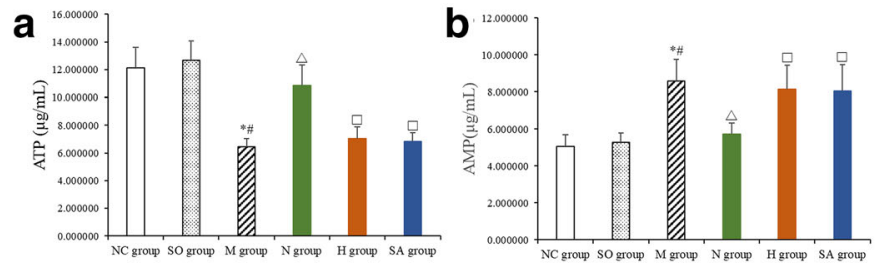

e
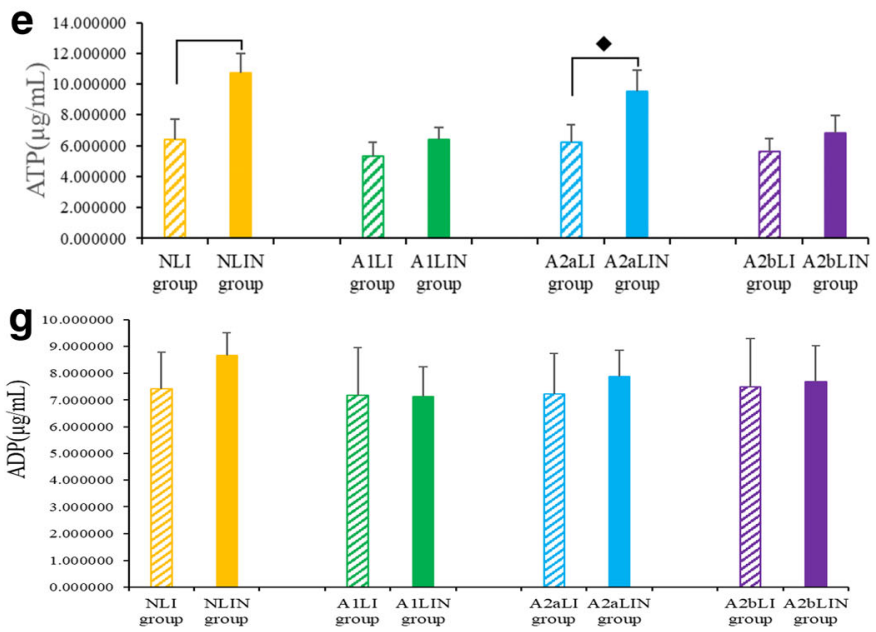

Fig. 3 Effects of EA on ATP, AMP, ADP, and ADO concentrations. The $\operatorname{ATP}(\mathbf{a}, \mathbf{e}), \operatorname{AMP}(\mathbf{b}, \mathbf{f}), \operatorname{ADP}(\mathbf{c}, \mathbf{g})$, and $\operatorname{ADO}(\mathbf{d}, \mathbf{h})$ levels in the cardiac tissue. Data are presented as mean \pm SD. $* P<0.01$ versus $\mathrm{NC}$ group,
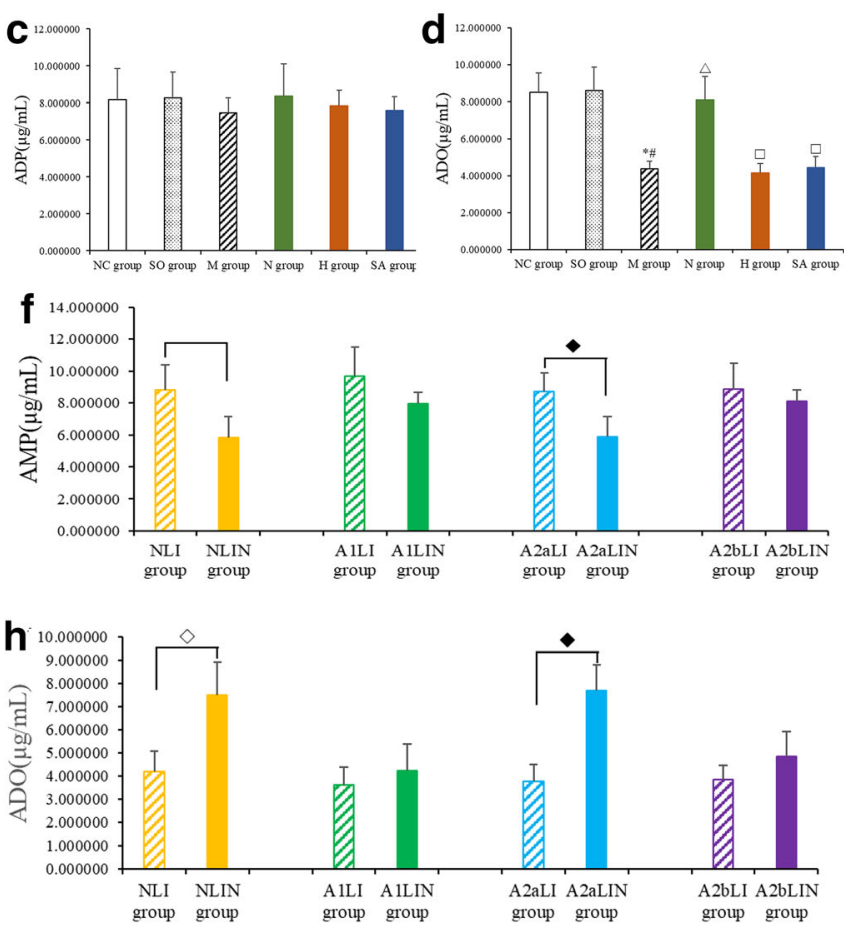

$\# P<0.01$ versus $\mathrm{SO}$ group, $\triangle P<0.01$ versus $\mathrm{M}$ group, $\diamond P<0.01$ versus NLI group, $\bullet P<0.01$ versus A2aLI group

and the increase of AMP. Previous studies indicated that recovery of myocardial function after $\mathrm{MI}$ is relevant to recovery of ATP [29], conversion of AMP to ADO has antiinflammatory effect [30], and ADO has function of regulating inflammatory by binding to adenosine receptors [31], thus, it is speculated that the effect of EA at PC6 may be related to the regulating effect of EA at PC6 in ATP, AMP, and ADO.

Knockdown of A1AR or A2bAR abolished EA at PC6induced functional protection via affecting RPP and ECG. Moreover, knockdown of A1AR and A2bAR reversed influences of ATP, AMP, and ADO production caused by EA at PC6. The cardio protection of adenosine receptor needs activating other adenosine receptors [32-35]. In our results, knockdown of A1AR or A2bAR reversed upregulation of the other two adenosine receptor expression levels and knockdown of A2aAR could not abolish the influence of EA at PC6 in upregulating the expression levels of A1AR and A2bAR. These observations showed that cardio protection of EA requires activating $\mathrm{A} 1 \mathrm{AR}$ and $\mathrm{A} 2 \mathrm{bAR}$, but not $\mathrm{A} 2 \mathrm{aAR}$, and indicated one of reasons for these results.

Activating adenosine receptors can improve MI [36, 37]. For instance, activated A2aAR can reduce cardiac mast cell degranulation and protect ischemic myocardium [38]; A2bAR agonist has effect on improving MI injury, and this effect depends on activation of protein kinase C [37]. Previous experiment also detected the expression levels of A1AR, A2aAR, and A2bAR of MI rats after EA treatment [22]; both 

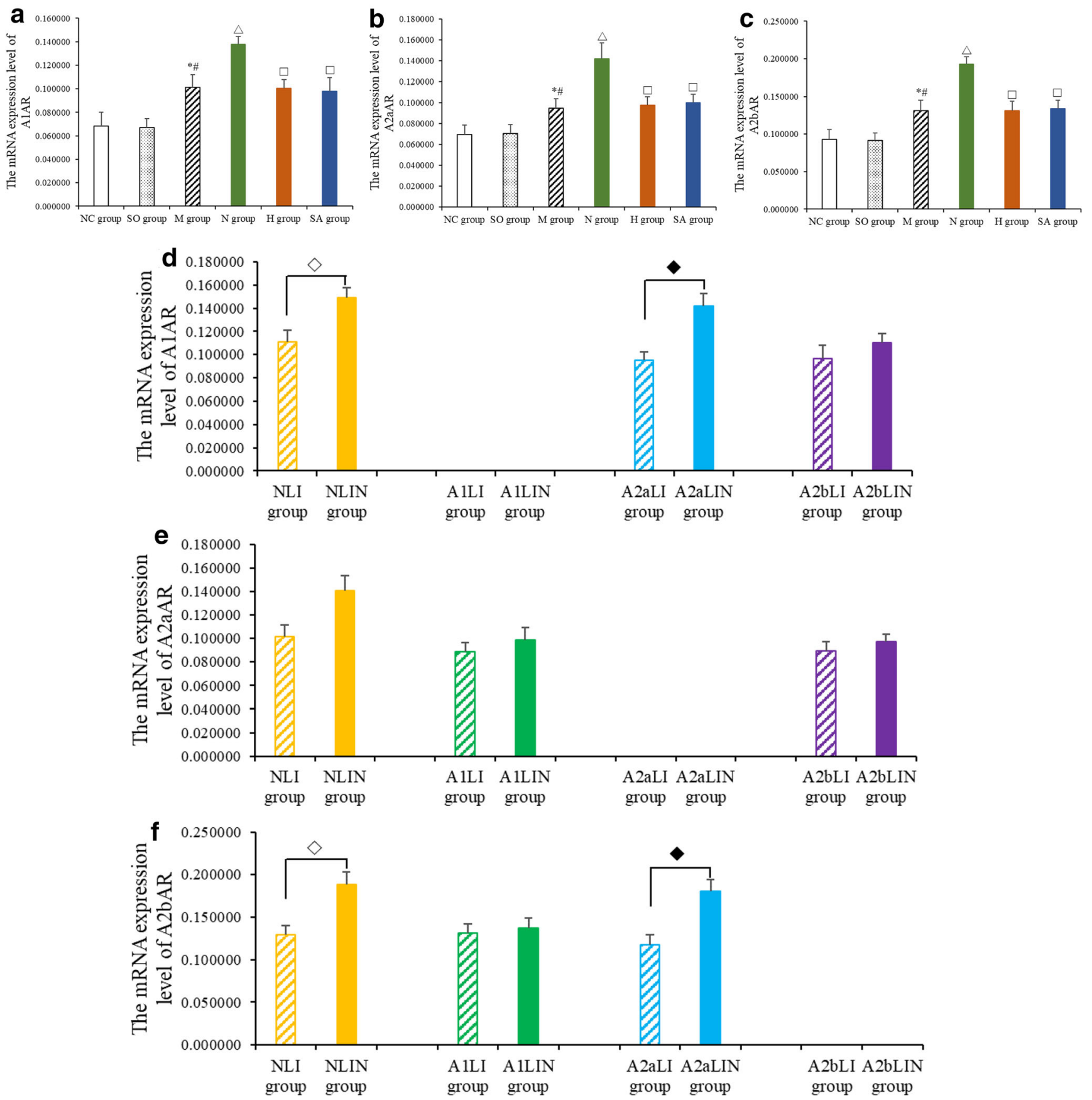

Fig. 4 Effects of EA on the mRNA expression levels of A1AR, A2aAR, and A2bAR. The mRNA expression levels of A1AR (a, d), A2aAR (b, e), A2bAR (c, f) detected by in situ hybrization (ISH). Data are presented

studies agreed that EA at PC6 could effectively upregulate the expression levels of A2aAR and A2bAR. On account of the differences in EA treatment protocol and detection method for the expression levels of adenosine receptors, there were disagreements about whether EA at PC6 could affect the expression level of A2aAR. Considering the upregulation of adenosine receptors is not equated with the activation of adenosine receptors, we knockdown the expression level of adenosine receptors. After silence of A1AR or A2bAR, EA at PC6 could as mean $\pm \mathrm{SD}$. $* P<0.01$ versus $\mathrm{NC}$ group, $\# P<0.01$ versus $\mathrm{SO}$ group, $\triangle P<0.01$ versus M group, $\diamond P<0.01$ versus NLI group, $\diamond P<0.01$ versus A2aLI group

not improve MI injury, and after silence of A2aAR, EA at PC6 still attenuate MI injury. Thus, the cardio protection of EA at PC6 needs A1AR and A2bAR.

The protective effect of A1AR, A2aAR, and A2bAR needs other adenosine receptors, namely only one adenosine receptor cannot play its role. A2aAR and A2bAR must be simultaneously activated for cardiac protection to occur [33]. The antagonism of $\mathrm{A} 2 \mathrm{aAR}$ and $\mathrm{A} 2 \mathrm{bAR}$ can block the protective effect of A1AR [34]. Other previous experiments also showed 
that the cardiac protection of A1AR needs the activation of $\mathrm{A} 2 \mathrm{aAR}$ and $\mathrm{A} 2 \mathrm{bAR}[32,35]$. In these results, after the silence of A1AR or A2bAR, EA at PC6 could not upregulate the expression levels of other two adenosine receptors, and after the silence of A2aAR, EA at PC6 still upregulate the expression levels of A1AR and A2bAR. It may be one of reasons why the effect of EA at PC6 in improving MI depends on $\mathrm{A} 1 \mathrm{AR}$ and $\mathrm{A} 2 \mathrm{bAR}$, not A2aAR.

\section{Conclusion}

In conclusion, this study indicated that EA at PC6 is effective in alleviating MI injury, EA at PC6 is more effective than EA at LI6 and sham acupoint; after silence of A1AR or A2bAR, EA at PC6 could not improve MI injury; thus, the effect of EA at PC6 may depend on A1AR and A2bAR. In addition, one of the reasons may be that after the silence of A1AR or A2bAR, EA at PC6 could not impact the expression levels of the other two adenosine receptors, and after the silence of A2aAR, EA at PC6 could impact the expression levels of A1AR and A2bAR.

Authors' contributions Yulan Ren and Zhihan Chen contributed equally to this study. Conceived and designed the experiments: Yulan Ren. Performed the experiments: YulanRen, Zhihan Chen, Rui Wang, Yang $\mathrm{Yu}$, Dehua Li, Yonggang He. Analyzed the data: Zhihan Chen, Rui Wang, Yang Yu, Dehua Li. Wrote the paper: YulanRen, Zhihan Chen.

Funding information This study was supported by grants from the National Natural Science Foundation of China (81373561).

Data availability Not applicable.

\section{Compliance with ethical standards}

Conflict of interest The authors declare that they have no conflict of interest.

Ethics approval This experiment was approved by ethical committee of Chengdu University of Traditional Chinese Medicine (no. 2014-03).

Consent to participate Not applicable.

Consent for publication All authors read the final manuscript and agreed to publish the manuscript.

Code availability Not applicable.

Open Access This article is licensed under a Creative Commons Attribution 4.0 International License, which permits use, sharing, adaptation, distribution and reproduction in any medium or format, as long as you give appropriate credit to the original author(s) and the source, provide a link to the Creative Commons licence, and indicate if changes were made. The images or other third party material in this article are included in the article's Creative Commons licence, unless indicated otherwise in a credit line to the material. If material is not included in the article's Creative Commons licence and your intended use is not permitted by statutory regulation or exceeds the permitted use, you will need to obtain permission directly from the copyright holder. To view a copy of this licence, visit http://creativecommons.org/licenses/by/4.0/.

\section{References}

1. Heusch G (2016) Myocardial ischemia: lack of coronary blood flow or myocardial oxygen supply/demand imbalance? Circ Res 119: 194-196

2. World Health Organization (2018) The top 10 causes of death. Available from https://www.who.int/en/news-room/fact-sheets/ detail/the-top-10-causes-of-death

3. The Joint Task Force for Guideline on the Assessment and Management of Cardiovascular Risk in China (2019) Guideline on the assessment and Management of Cardiovascular Risk in China. Chinese Circulation Journal 34:4-28

4. Viera AJ, Rietz A (2017) Ischemic heart disease. Fam Med:973981

5. Fihn SD, Gardin JM, Abrams J et al (2012) 2012 ACCF/AHA/ ACP/AATS/PCNA/SCAI/STS guideline for the diagnosis and Management of Patients with Stable Ischemic Heart Disease. J Am Coll Cardiol 60:e44-e164

6. Committee of experts on rational drug use national health and family planning commission of the P.R.China, Chinese pharmacists association (2018) Medication Guide for Coronary Heart Disease (second edition). Chinese Journal of the Frontiers of Medical Science (Electronic Version) 10: 1-130

7. Saleh AI, Abdel Maksoud SM, SA EI-M et al (2011) Protective effect of L-arginine in experimentally induced myocardial ischemia: comparison with aspirin. J Cardiovasc Pharmacol Ther 16: 53-62

8. Pragani MA, Desai KP, Morrone D et al (2017) The role of nitrates in the management of stable ischemic heart disease: a review of the current evidence and guidelines. Rev Cardiovasc Med 18:14-20

9. Fihn SD, Blankenship JC, Alexander KP et al (2014) 2014 ACC/ AHA/AATS/PCNA/SCAI/STS focused update of the guideline for the diagnosis and management of patients with stable ischemic heart disease. J Am Coll Cardiol 1:1-35

10. Abdelnaby MH (2018) Effect of percutaneous coronary intervention on heart rate variability in coronary artery disease patients. European Cardiology:60-61

11. Dominiak M, Drożdż J (2009) Coronary artery bypass grafting operation is an optimal therapy in patients with high stage multivessel coronary artery heart disease. Polish journal of cardiothoracic surgery 6:181-186

12. Zhao L, Li D, Zheng $\mathrm{H}$ et al (2019) Acupuncture as adjunctive therapy for chronic stable angina. JAMA Intern Med 179:e1-e12

13. Liu L, Wang S, Fu C et al (2016) Comparison of beneficial effects of acupuncture at PC6, LU7 \& non-acupuncture points on cardiac cell function in myocardial ischemia (MI) rats by testing protein expression of L-type calcium channel protein and its related protein. Acupunct Electrother Res 41:107-125

14. Wang S, Ren L, Jia L et al (2015) Effect of acupuncture at Neiguan (PC 6) on cardiac function using echocardiography in myocardial ischemia rats induced by isoproterenol. J Tradit Chin Med 35:653658

15. Wang Y, Wang W, Li D et al (2014) The beneficial effect of electro-acupuncture given at PC6 (Neiguan-point) by the increase in cardiac transient outward $\mathrm{K}+$ current channel which depends on the gene and protein expressions in artificially induced myocardial ischemia rats. Acupunct Electrother Res 39:259-273 
16. He D, Ren Y, Tang Y et al (2013) Analysis of characteristics of meridians and acupoints selected for modern acu-moxibustion treatment of angina pectoris based on data mining. Liaoning Journal of Traditional Chinese Medicine 40:2195-2197

17. Ballegaard S, Meyer CN, Trojaborg W (1991) Acupuncture in angina pectoris: does acupuncture have a specific effect? J Intern Med 229:357-362

18. Ballegaard S, Jensen G, Pedersen F et al (1986) Acupuncture in severe, stable angina pectoris: a randomized trial. Acta Med Scand 220:307-313

19. Wang Y, Zhang XL, Wang W et al (2015) The beneficial effects of electro-acupuncture at PC6 (Neiguan-point) of gene and protein expressions of classical inward-rectifier potassium channels in myocardial ischemic rats. Acupunct Electrother Res 40:335-353

20. Cheng ZD, Li CR, Shao XJ et al (2013) The impacts of alongchannel acupuncture on the protein expressions of the chloride channel of the rats with myocardial ischemia. Evid Based Complement Alternat Med 2013:321067

21. Du T (2017) Acupuncture at Neiguan for myocardial ischemia in rats: cardioprotective and purine signaling mechanism study. Chengdu Univerisity of Traditional Chinese Medicine

22. Lu SF, Tang YX, Ding YJ et al (2018) Effects of electroacupuncture on the expression of adenosine receptors in the heart tissue of myocardial ischemia rats. Chinese Acupuncture \& Moxibustion 38:173-178

23. Yu SG, Xu B, Chen RX, et al (2016) Experimental Acupuncture Science. People's Medical Publishing House

24. Wang JY (2010) Effect of acupuncture at Taichong (LR3) and nonacupoint on the blood pressure and medulla protein expression in spontaneously hypertensive rats. Guangzhou University of Chinese Medicine

25. Imahashi K, Pott C, Goldhaber JI et al (2005) Cardiac-specific ablation of the $\mathrm{Na}^{+}-\mathrm{Ca}^{2+}$ exchanger confers protection against ischemia/reperfusion injury. Circ Res:916-921

26. Jennings RB, Steenbergen C Jr (1985) Nucleotide metabolism and cellular damage in myocardial ischemia. Annu Rev Physiol 47: 727-749

27. Tang Y, Yin HY, Rubini P et al (2016) Acupuncture-induced analgesia: a neurobiological basis in purinergic signaling. Neuroscientist 22:563-578
28. Gordon JL (1986) Extracellular ATP: effects, sources and fate. Biochem J 233:309-319

29. Pasque MK, Wechsler AS (1984) Metabolic intervention to affect myocardial recovery following ischemia. Ann Surg 200:1-12

30. Shin EY, Wang L, Zemskova M et al (2017) Adenosine production by biomaterial-supported mesenchymal stromal cells reduces the innate inflammatory response in myocardial ischemia/reperfusion injury. J Am Heart Assoc 7:e006949

31. Chen L, Fredholm BB, Jondal M (2008) Adenosine, through the A1 receptor, inhibits vesicular MHC class I cross-presentation by resting DC. Mol Immunol 45:2247-2254

32. Urmaliya VB, Pouton CW, Ledent C et al (2010) Cooperative cardioprotection through adenosine $\mathrm{A} 1$ and $\mathrm{A} 2 \mathrm{a}$ receptor agonism in ischemia-reperfused isolated mouse heart. J Cardiovasc Pharmacol 56:379-388

33. Methner C, Schmidt K, Cohen MV et al (2010) Both A2a and A2b adenosine receptors at reperfusion are necessary to reduce infarct size in mouse hearts. Am J Physiol Heart CircPhysiol 299:H1262H1264

34. Lasley RD, Kristo G, Keith BJ et al (2007) The A2a/A2b receptor antagonist ZM-241385 blocks the cardioprotective effect of adenosine agonist pretreatment in in vivo rat myocardium. Am J Physiol Heart Circ Physiol 292:H426-H431

35. Zhan E, Mcintosh VJ, Lasley RD (2011) Adenosine A2A and A2B receptors are both required for adenosine A1 receptor-mediated cardioprotection. Am J Physiol Heart Circ Physiol 301:H1183H1189

36. Mcintosh VJ, Lasley RD (2012) Adenosine receptor-mediated cardioprotection are all 4 subtypes required or redundant. J Cardiovasc Pharmacol Ther 17:21-33

37. Philipp S, Yang XM, Cui L et al (2006) Postconditioning protects rabbit hearts through a protein kinase $\mathrm{C}$-adenosine $\mathrm{A} 2 \mathrm{~b}$ receptor cascade. Cardiovasc Res 70:308-314

38. Rork TH, Wallace KL, Kennedy DP et al (2008) Adenosine A2A receptor activation reduces infarct size in the isolated, perfused mouse heart by inhibiting resident cardiac mast cell degranulation. Am J Physiol Heart Circ Physiol 295:H1825-H1833

Publisher's note Springer Nature remains neutral with regard to jurisdictional claims in published maps and institutional affiliations. 